\title{
A circuit for measuring the gap voltage of a scanning tunneling microscope on a nanosecond time scale
}

\author{
M. Ochmann, ${ }^{\text {a) }}$ H.-J. Münzer, J. Boneberg, and P. Leiderer \\ Universität Konstanz, Fakultät für Physik, D-78457 Konstanz, Germany
}

(Received 20 October 1998; accepted for publication 15 December 1998)

\begin{abstract}
We demonstrate a new circuit design for fast measurements of the voltage drop across the gap of a scanning tunneling microscope (STM) based on the simultaneous operation of two different amplifiers. The first is a fast instrumentation amplifier, sensing directly the voltage drop across the tunneling barrier, the second is a medium speed current amplifier with an overall gain of $10^{8} \mathrm{~V} / \mathrm{A}$, suitable for normal STM operation. We obtained a time resolution of $10 \mathrm{~ns}$ measuring the plasma ignition under a STM tip during illumination with an intense $10 \mathrm{~ns}$ laser pulse. Possible applications include the study of STM point contacts. (C) 1999 American Institute of Physics.

[S0034-6748(99)00504-3]
\end{abstract}

\section{INTRODUCTION}

The temporal response of scanning probe microscopes (SPMs) is usually limited to a few milliseconds. On the other hand, technology steps towards device dimensions far below $100 \mathrm{~nm}$ and operating speed beyond GHz. Thus, the combination of high lateral and temporal resolution in SPM becomes more and more important. Recently, several groups have addressed this problem. One approach is the use of short laser pulses in a pump-probe setup, utilizing nonlinearities in the tip-sample interaction [for example, the $I V$ curve of a scanning tunneling microscope (STM) or the force-distance characteristic of an atomic force microscope $(\mathrm{AFM})]{ }^{1,2}$ Microwaves have been used instead of pulsed lasers, too. ${ }^{3-6}$ Finally, inserting photoconductive switches into the signal path in connection with pump-probe techniques can provide high time resolution as well. ${ }^{7,8}$ With these methods it is possible to achieve picosecond time resolution.

For many applications such an ultimate speed is not necessary and, therefore, the expense of the pump-probe methods is not justified. Additionally, the pump-probe technique is not much useful, if the signal to be measured is not repetitive. Thus, if one wants to record the complete time response of the system for each single shot, pure electronic measurement techniques are preferable. For example, there has recently been a discussion about the mechanism of the socalled FOLANT technique (short for "focusing of laser radiation in the near field of a tip"), which was proposed as a method for nanostructuring of several different materials. ${ }^{9-13}$ Thereby the tip of a STM or AFM is illuminated with an intense nanosecond laser pulse. The relevant thermal and possibly field-induced processes responsible for the structuring are expected to occur on the time scale of the laser pulse. On the first sight the obvious way would be to look for any signature in the tunneling current, but the extremely high source impedances and small currents normally involved in scanning tunneling microscopy (usually in the

${ }^{a)}$ Electronic mail: michael.ochmann@uni-konstanz.de order of G $\Omega$ ) limit the bandwidth of any current amplifier to about $1 \mathrm{MHz} .{ }^{14-16}$ Indeed, one can observe large transient currents even with a slow amplifier, but it is difficult to decide which origin such signals may have. We, therefore, tried to measure directly the voltage drop across the tunneling gap with a commercial instrumentation amplifier, operated simultaneously to the current amplifier. With this arrangement we found quantization steps in the gap resistance, suggesting strongly that the current transients result from a mechanical tip-sample contact that occurs during the illumination due to a thermal expansion of the tip. ${ }^{17}$

The temporal resolution of these measurements was limited to about $1 \mu \mathrm{s}$, mainly because of the intrinsic bandwidth of the commercial instrumentation amplifier. In this article we present an improved version, built with discrete components, which allow us to increase the bandwidth considerably. In Sec. II we describe in detail the design and principle of operation of the amplifier and present in Sec. III some results, demonstrating its properties in measuring fast transient electronic signals with the STM.

\section{CIRCUIT DESIGN AND PERFORMANCE TESTS}

Figure 1 shows the principle of our combined amplification system. The tunneling resistance is depicted as a variable resistor, which is a function of the applied bias voltage, the selected tunneling current (we assume a constant-current operation mode), the electronic properties of tip and sample, and time.

We decided to use the "classical" scheme of current sensing: a $500 \mathrm{k} \Omega$ metal film resistor is placed in series with the tunneling contact and a noninverting amplifier with a gain of 200 is then used to amplify the voltage drop across the resistor. The overall gain of the circuit is approximately $10^{8} \mathrm{~V} / \mathrm{A}$. This configuration allows a current of $1 \mu \mathrm{A}$ to flow during a tip-sample contact at a bias voltage of $500 \mathrm{mV}$ (which is still convenient for a proper STM operation under ambient conditions), resulting in a voltage drop of approximately $25 \mathrm{mV}$ across the expected point contact resistance of 


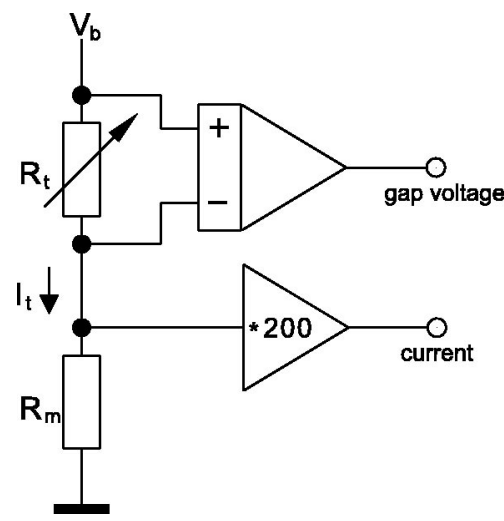

FIG. 1. Principle of the amplifier. An instrumentation amplifier measures the voltage drop across the tunneling resistance (depicted as variable resistor $R_{t}$ ). The lower part of the circuit is a common current amplifier used to operate the STM in constant-current mode.

$2 e^{2} / h=12.9 \mathrm{k} \Omega$. This voltage drop is sufficient to be sensed by the instrumentation amplifier which will be described below.

For the current amplifier (lower part of the circuit in Fig. 1) we choose the OPA637 operational amplifier (opamp) from Burr-Brown, because it combines high input impedance (up to $10^{12} \Omega$ ), low offset voltage (about $100 \mu \mathrm{V}$ ), and a unity-gain bandwidth of $80 \mathrm{MHz}$. Because of the high voltage gain the input offset voltage of the opamp and noise are much more a problem as with the transimpedance-type amplifier. ${ }^{14} \mathrm{~A}$ stable and precise offset voltage trimming is essential in this application.

We obtained an almost ideal low-pass frequency response with a cutoff frequency of $180 \mathrm{kHz}$. The amplifier's noise amounts to approximately $25 \mathrm{pA}_{\mathrm{pp}}$ within the bandwidth of the STM feedback loop (about $1 \mathrm{kHz}$ ). This is well suited for high-resolution topographic imaging.

We will now focus the discussion on the more important part of the circuit, the instrumentation amplifier. The basic demands for this device are similar to those mentioned above for the current amplifier: high input impedance to minimize current leakage out of the signal path, low offset voltage, low noise, and high speed. There are some excellent monolithic devices available which fulfill the first three points almost completely. We tested the INA110 from Burr-Brown, a JFET input, very precise monolithic instrumentation amplifier. With this device we conducted the gap resistivity measurements mentioned in the introduction. ${ }^{17}$ However, due to the limited response time (about $1 \mu \mathrm{s}$ ) it was not possible to make any statements about processes occurring on a faster time scale.

For this reason we applied the circuit depicted in Fig. 2, which is built around the OPA655, a $400 \mathrm{MHz}$ JFET opamp, and follows the classical scheme of a three-opamp instrumentation amplifier. This circuit is described in detail in the datasheet for the OPA655, ${ }^{18}$ thus we will only review some properties which are important for our special application. The differential gain of the amplifier is 1.5 when applied to an impedance matched $50 \Omega$ load, a fast digitizing oscilloscope in most cases. All resistors are $0.1 \%$ metal-film types resulting in a $2 \mathrm{mV}$ voltage offset. The useful input voltage

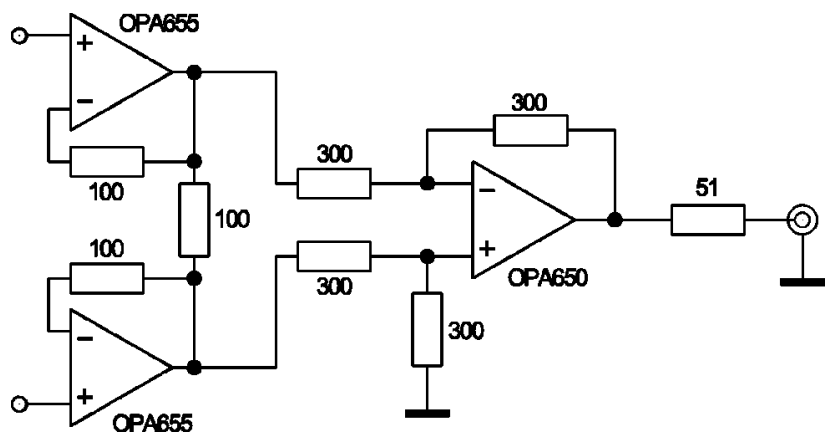

FIG. 2. Schematic diagram of the fast instrumentation amplifier. All resistor values are given in $\Omega$.

range is limited to about $\pm 0.9 \mathrm{~V}$, which is sufficient for STM operation on metals and most semiconductors under ambient conditions.

The voltage difference measured by the amplifier in the case of dc signals could be obtained by applying the formula for a voltage divider (Fig. 3):

$$
V_{\text {gap }}=V_{b} \frac{R_{t}}{R_{m}+R_{t}},
$$

with $V_{b}$ the bias voltage, $R_{m}$ the measuring resistor of $500 \mathrm{k} \Omega$, and $R_{t}$ the gap resistance. It should be noted that the instrumentation amplifier is naturally not sensitive in the case of very high tunneling resistances, because almost the complete bias voltage drops across the gap. Each of the opamp input terminals exhibits a small but not negligible capacity, depicted in Fig. 3 as $C_{1}$ and $C_{m}$, respectively. Since $C_{1}$ is driven by the low impedance voltage source $V_{b}$ it can be neglected for the following calculation. $C_{m}$ is dominated by the input capacity of the opamp, which is in the order of $1 \mathrm{pF}$. The second important capacity $C_{t}$ in parallel to the tunneling resistor is a sum of the geometric gap capacity ${ }^{7}$ $C_{g}$ and the stray capacity $C_{s}$ of the instrumentation amplifier. The capacity of the tunneling junction $C_{g}$, typically in the order of $10^{-3} \mathrm{pF}$, can be neglected, but not $C_{s}$, which amounts to approximately $0.1 \mathrm{pF}$.

The absolute value of the voltage difference measured between the input terminals of the instrumentation amplifier is then given by

$$
V_{\text {gap }}=\left|V_{b} \frac{R_{t} \| 1 / i \omega C_{t}}{R_{t}\left\|1 / i \omega C_{t}+R_{m}\right\| 1 / i \omega C_{m}}\right| .
$$

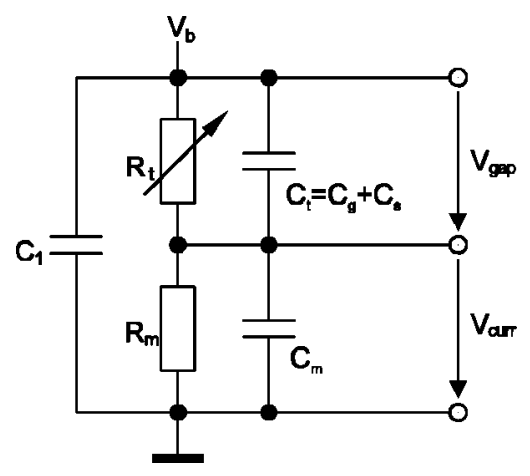

FIG. 3. High-frequency equivalent circuit of the system. Typical values for the tunneling resistance $R_{t}$ are between $\mathrm{k} \Omega$ and $\mathrm{G} \Omega$. 


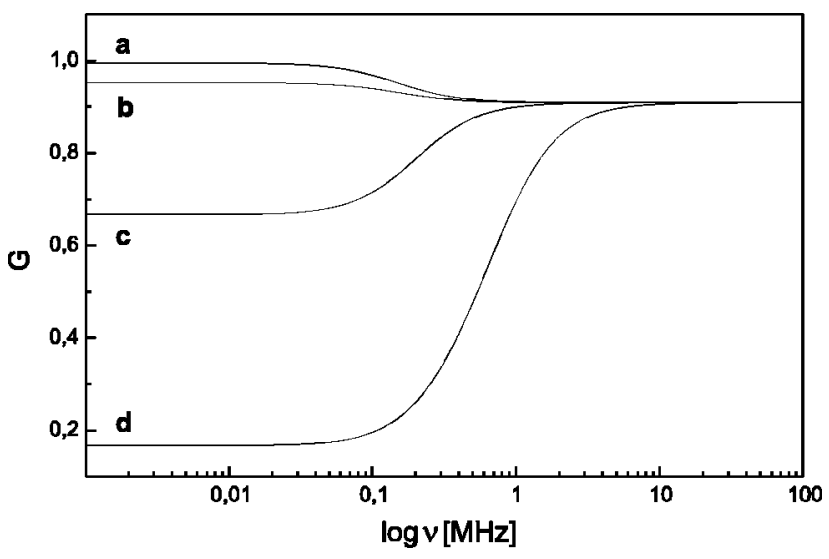

FIG. 4. Calculated frequency response for several combinations of $R_{t} / R_{m}$. The curves are essentially flat for high frequencies. (a) $R_{t}=100 \mathrm{M} \Omega$, (b) $R_{t}=10 \mathrm{M} \Omega$, (c) $R_{t}=1 \mathrm{M} \Omega$, and (d) $R_{t}=100 \mathrm{k} \Omega . C_{t} / C_{m}=0.1$ and $R_{m}$ $=500 \mathrm{k} \Omega$.

[The symbol $\|$ denotes the parallel resistance, i.e., $R_{1} \| R_{2}$ means $\left.R_{1} R_{2} /\left(R_{1}+R_{2}\right)\right]$. After some simple algebra one obtains the transfer function of the circuit:

$$
G(\omega)=\frac{V_{\text {gap }}}{V_{b}}=\frac{R_{t}}{R_{t}+R_{m}} \sqrt{\frac{1+\frac{\omega^{2}}{\omega_{m}^{2}}}{1+\frac{\omega^{2}}{\omega_{m}^{2}} \frac{\left(1+C_{t} / C_{m}\right)^{2}}{\left(1+R_{m} / R_{t}\right)^{2}}}},
$$

with $\omega_{m}=1 / R_{m} C_{m}$. This expression reveals a very interesting property: If we choose the ratio of the capacities equal to the resistivities, i.e., $C_{t} / C_{m}=R_{m} / R_{t}$, we obtain a complete frequency compensation of the circuit as described by the pure resistive voltage divider in Eq. (1). Although not applicable in our experiments, where $R_{t}$ varies essentially, one may "trim" the frequency response of the amplifier for a specific tunneling resistance to every desired flatness.

Figure 4 shows $G(\omega)$ for several values of $R_{t}$ (and $C_{t} / C_{m}=0.1$ as explained above). In all cases we find an almost flat high-frequency response. Thus, the bandwidth of the whole circuit is only limited by the intrinsic bandwidth of the instrumentation amplifier. In particular, there is no gain peaking, which improves the step response of the amplifier considerably (see Sec. III).

We installed our amplifier within a home-built STM with optical access. The complete amplifier resides inside the base of the STM, therefore, all necessary cables are not longer than approximately $2 \mathrm{~cm}$.

\section{RESULTS}

The bandwidth of the instrumentation amplifier alone was found to be slightly above $100 \mathrm{MHz}$. In order to test the time resolution of the complete circuit and to give a possible application of the device we looked for a physical process that shows the capabilities of the amplifier in situ, i.e., inside the STM environment, and occurs on the smallest possible time scale. Thus, we focused pulses of a frequency-doubled $\mathrm{Nd}$ :YAG laser at $532 \mathrm{~nm}$ with an energy of about $80 \mathrm{~mJ} /$ pulse and $10 \mathrm{~ns}$ duration into the gap region. The tip (an

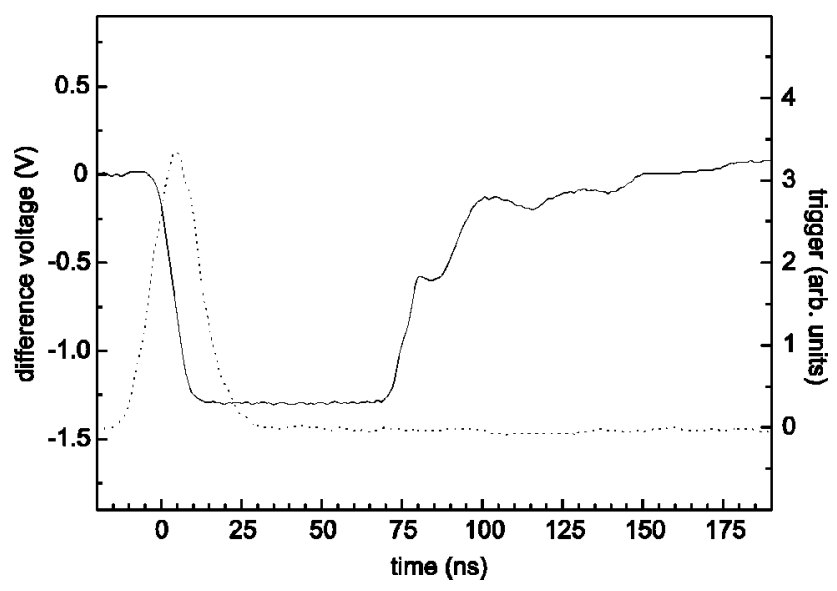

FIG. 5. Time response (solid line) of the instrumentation amplifier measured with a plasma ignition. The slew rate is in the order of $100 \mathrm{mV} / \mathrm{ns}$ for a full scale signal. The laser pulse is shown as dotted line for comparison.

electrochemically etched tungsten wire) was retracted by about $0.1 \mathrm{~mm}$ against a copper plate and held at ground potential. A plasma ignition could be clearly observed visually. Figure 5 shows a typical example of the signals we observed. Despite the obviously very complicated processes involved in such a plasma ignition, the amplifier exhibits a slew rate of roughly $100 \mathrm{mV} / \mathrm{ns}$ for a full scale signal and no significant ringing or overshots. This agrees well with the bandwidth calculation in the last section. Additionally, the device has proven to be very useful for fast measurements of the thermal expansion of the tip under tunneling conditions, showing essentially the same time resolution as stated above. $^{19}$

As a second application of our device we tried to reproduce the point contact measurements observed with the INA110 as described in the last section. Indeed, we found the quantization steps as shown in Fig. 6 with a mechanically cut silver tip on a gold substrate at $U_{t}=-700 \mathrm{mV}$. This behavior is well known to occur during the breaking of a metallic point contact. ${ }^{20-23}$ Although the input properties of the discrete setup are clearly not as precise as the monolithic,

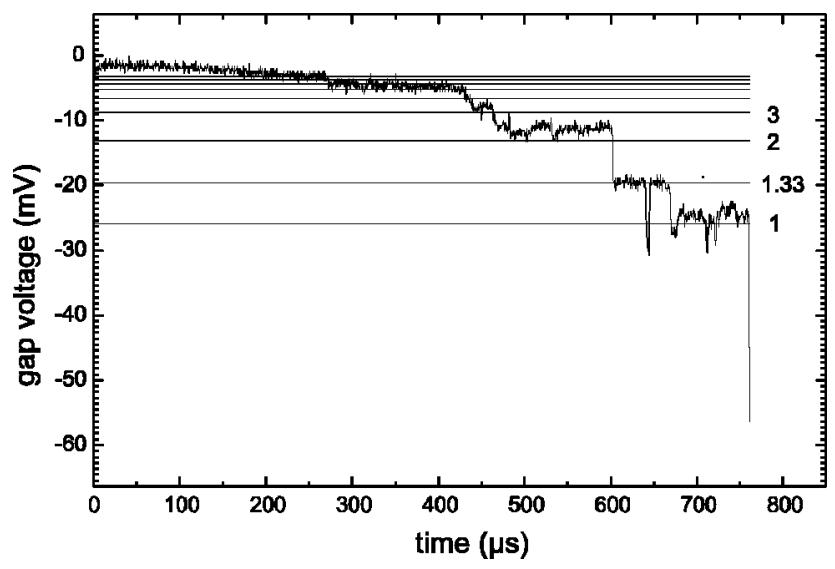

FIG. 6. Breaking of a metallic point contact: quantized resistance steps measured during the retraction of the STM tip. The horizontal lines indicate the resistance values of $12.9 \mathrm{k} \Omega / n$. The numbers on the right give the corresponding $n$. $V_{b}=-700 \mathrm{mV}$. 
we obtained a resistance resolution of about $1 \mathrm{k} \Omega$ limited only by noise.

\section{ACKNOWLEDGMENT}

This work was supported by the Deutsche Forschungsgemeinschaft (SFB513).

${ }^{1}$ G. Nunes and M. R. Freeman, Science 262, 1029 (1993).

${ }^{2}$ G. M. Steeves, A. Y. Elezzabi, and M. R. Freeman, Appl. Phys. Lett. 72, 504 (1998).

${ }^{3}$ A. A. Lucas, P. H. Cutler, T. E. Feuchtwang, T. T. Tsong, T. E. Sullivan, Y. Tuk, H. Nguyen, and P. J. Silverman, J. Vac. Sci. Technol. A 6, 461 (1988).

${ }^{4}$ L. Arnold, W. Krieger, and H. Walter, Phys. Rev. B 41, 10229 (1990).

${ }^{5}$ W. Mizutani, B. Michel, R. Scherer, H. Wolf, and H. Rohrer, Appl. Phys. Lett. 63, 147 (1993).

${ }^{6}$ S. J. Strabick and P. S. Weiss, Rev. Sci. Instrum. 65, 918 (1993).

${ }^{7}$ S. Weiss, D. Botkin, D. F. Ogletree, M. Salmeron, and D. S. Chemla, Phys. Status Solidi B 188, 342 (1995).

${ }^{8}$ U. D. Keil, J. R. Jensen, and J. M. Hvarn, J. Appl. Phys. 81, 2929 (1997).

${ }^{9}$ J. Jersch and K. Dickmann, Appl. Phys. Lett. 68, 868 (1996).
${ }^{10}$ J. Jersch, F. Demming, L. J. Hildenhagen, and K. Dickmann, Appl. Phys. A: Mater. Sci. Process. 66A, 29 (1998)

${ }^{11}$ V. A. Ukraintsev and J. T. Yates, Jr., J. Appl. Phys. 80, 2561 (1996).

${ }^{12}$ I. Lyubinetsky, Z. Dohnálek, V. A. Ukraintsev, and J. T. Yates, Jr., J. Appl. Phys. 82, 4115 (1997).

${ }^{13}$ J. Boneberg, M. Tresp, M. Ochmann, H.-J. Münzer, and P. Leiderer, Appl. Phys. A: Mater. Sci. Process. 66A, 615 (1998).

${ }^{14}$ J. Graeme, Photodiode Amplifiers (McGraw-Hill, New York, 1996).

${ }^{15}$ H. Birk, K. Oostveen, and C. Schönenberger, Rev. Sci. Instrum. 67, 2977 (1996).

${ }^{16}$ U. Dürig, L. Novotny, and B. Michel, Rev. Sci. Instrum. 68, 3814 (1997).

${ }^{17}$ J. Boneberg, H.-J. Münzer, M. Tresp, M. Ochmann, and P. Leiderer, Appl. Phys. A: Mater. Sci. Process. 67A, 381 (1998).

${ }^{18}$ Burr-Brown Corporation, OPA655-Wideband FET-Input Operational Amplifier (1996).

${ }^{19}$ (Unpublished).

${ }^{20}$ J. I. Pascual, J. Méndez, J. Gómez-Herrero, A. M. Baró, N. Garcia, U. Landmann, W. D. Luedtke, E. N. Bogachek, and H.-P. Cheng, Science 267, 1795 (1995).

${ }^{21}$ J. M. Krans, J. M. van Rultenbeek, V. V. Flans, I. K. Yanson, and L. J. de Jongh, Nature (London) 375, 767 (1995).

${ }^{22}$ J. A. Torres and J. J. Senz, Phys. Rev. Lett. 77, 2245 (1996).

${ }^{23}$ I. P. Batra, Surf. Sci. 395, 43 (1998). 